\title{
Fractal diffusion retrospective problems
}

\section{O. Yaremko*1}

\section{Received $6^{\text {th }}$ October 2013, Accepted $24^{\text {th }}$ January 2014}

Abstract: In this article we study the retrospective inverse problem. The retrospective inverse problem consists of in the reconstruction of a priori unknown initial condition of the dynamic system from its known final condition. Existence and uniqueness of the solution is proved.

Keywords: Hermite functions, retrospective problem, integral equation, fractal diffusion.

\section{Introduction}

In this article we study the retrospective inverse problem. The retrospective Inverse problem consists of in the reconstruction of a priori unknown initial condition of the dynamic system from its known final condition. The direct problem of heat conductivity is well-posed; the inverse problem is not well-posed. In mathematics the vast majority of inverse problems set not wellposed - small perturbations of the initial data (observations) can correspond to arbitrarily large perturbations of the solution. The French mathematician Jacques Hadamard in 1939 defined, the problem is called correct or well-posed problem if a solution exists, the solution is unique, the solution's behavior hardly changes when there's a slight change in the initial condition. If at least one of these three conditions is not fulfilled, problems are termed ill-posed or not well-posed. The most often in the case of ill-posed problems of the third condition are violated the condition of the stability of solutions. In this case, there is a paradoxical situation: the problem is mathematically generated, but the solution cannot be obtained by conventional methods. A classic example of ill-posed problem is retrospective problem for heat equation on the real axis. Mathematically retrospective problem leads to a Fredholm integral equation of the first kind:

$\int_{-\infty}^{\infty} \frac{1}{2 \sqrt{\pi \tau}} e^{\left(-\frac{(x-\xi)^{2}}{4 \tau}\right)} \hat{f}(\xi) d \xi=\hat{u}(\tau, x)$,

in which $\hat{f}(x)$ - is the initial distribution of the temperature

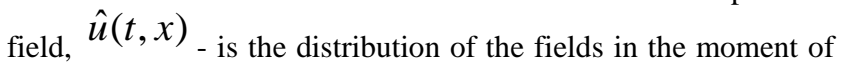
time t. As shown in [1], the solution of equation (1) expressed by the formula:

$$
\hat{f}(x)=\frac{1}{\sqrt{\pi}} \sum_{j=0}^{\infty} \frac{\hat{u}^{(j)}(0)}{(2 \sqrt{\tau})^{n+1} j !} H_{j}\left(\frac{x}{2 \sqrt{\tau}}\right) .
$$

\section{Problem Statement}

In the inverse problem of heat conductivity the initial distribution of sources is unknown. The initial distribution of sources generates the specified temperature distribution in an infinite piecewise-homogeneous rod In. Mathematical statement of the problem consists in finding a solution separatist system $(n+1)$ equations of parabolic type

$$
\left(\frac{\partial}{\partial t}-a_{j}^{2} \frac{\partial^{2}}{\partial x^{2}}\right) u_{j}(t, x)=0, t>0, x \in I_{n},
$$

on the initial conditions

$\left.u_{j}(t, x)\right|_{t=0}=f_{j}(x), x \in I_{n}$,

on the boundary conditions

$\left.u_{1}\right|_{x=-\infty}=0,\left.u_{n+1}\right|_{x=\infty}=0$

and the conjugation conditions

$\left[\alpha_{m 1}^{k} \frac{\partial}{\partial x}+\beta_{m 1}^{k}\right] u_{k}=\left[\alpha_{m 2}^{k} \frac{\partial}{\partial x}+\beta_{m 2}^{k}\right] u_{k+1}$,

(6)

$x=l_{k}, k=1, \ldots, n ; m=1,2$,

here $u(t, x)$ - unknown function, $f(x)$ - set function,

$u(\tau, x)=\sum_{k=2}^{n} \theta\left(x-l_{k-1}\right) \theta\left(l_{k}-x\right) u_{k}(\tau, x)+$

$+\theta\left(l_{1}-x\right) u_{1}(\tau, x)+\theta\left(x-l_{n}\right) u_{n+1}(\tau, x)$,

$f(x)=\sum_{k=2}^{n} \theta\left(x-l_{k-1}\right) \theta\left(l_{k}-x\right) f_{k}(x)+$

$+\theta\left(l_{1}-x\right) f_{1}(x)+\theta\left(x-l_{n}\right) f_{n+1}(x)$ 
$\alpha_{m i}^{k}, \beta_{m i}^{k}, \gamma_{m i}^{k}, \delta_{m i}^{k}$

given real number, in which the condition of unlimited solvability of the problem considered fulfilled [2].

The solution to problem (3)-(6) is of the form:

$u_{k}(t, x)=\sum_{s=1}^{n+1} \int_{l_{s}}^{l_{s+1}} H_{k s}(t, x, \xi) f_{s}(\xi) d \xi$

where $H_{k s}(t, x, \xi) k, s=1, \ldots, n+1$ - influence function [2] of the mixed boundary value problem.

Retrospectiveproblemfortheheatequationinthecaseofinfinitepiece wise-homogeneous rod consists in the determination of the unknown initial distribution of sources $f(x)$, which generates the specified temperature distribution $u(\tau, x)$ in the moment of time $t=\tau$.

\section{Transformation operators}

Method of transformation operators is used to solve the problem [2]. Necessary definitions from [2]. The direct $J: f \rightarrow f \mathrm{f}$ and inverse $J^{-1}: f \rightarrow f$ transformation operators are set equalities:

$$
\begin{aligned}
& f(x)=\int_{-\infty}^{\infty} \varphi(x, \lambda)\left(\int_{-\infty}^{\infty} e^{-i \lambda \xi} \hat{f}(\xi) d \xi\right) d \lambda, \\
& \hat{f}(x)=\int_{-\infty}^{\infty} e^{-i \lambda \xi}\left(\int_{-\infty}^{\infty} \varphi^{*}(\xi, \lambda) f(\xi) d \xi\right) d \lambda .
\end{aligned}
$$

Here $\varphi(x, \lambda), \varphi^{*}(x, \lambda)$-are the eigenfunctions of the direct and conjugate Sturm-Liouville problems for the Fourier operator in piecewise-homogeneous axis In. Eigenfunction

$$
\begin{array}{r}
\varphi(x, \lambda)=\sum_{k=2}^{n} \theta\left(x-l_{k-1}\right) \theta\left(l_{k}-x\right) \varphi_{k}(x, \lambda)+ \\
\quad+\theta\left(l_{1}-x\right) \varphi_{1}(x, \lambda)+\theta\left(x-l_{n}\right) \varphi_{n+1}(x, \lambda)
\end{array}
$$

is a solution of the system of separate differential equations

$$
\left(a_{m}^{2} \frac{d^{2}}{d x^{2}}+\lambda^{2}\right) \varphi_{m}(x, \lambda)=0, x \in\left(l_{m}, l_{m+1}\right) ; m=1, \ldots, n+1,
$$

on the conjugation conditions

$$
\left[\alpha_{m 1}^{k} \frac{d}{d x}+\beta_{m 1}^{k}\right] \varphi_{k}=\left[\alpha_{m 2}^{k} \frac{d}{d x}+\beta_{m 2}^{k}\right] \varphi_{k+1},
$$

on the boundary conditions

$$
\left.\varphi_{1}\right|_{x=-\infty}=0,\left.\varphi_{n+1}\right|_{x=\infty}=0
$$

Similarly eigenfunction

$$
\begin{aligned}
& \varphi^{*}(\xi, \lambda)=\sum_{k=2}^{n} \theta\left(\xi-l_{k-1}\right) \theta\left(l_{k}-\theta\right) \varphi^{*}(\xi, \lambda)+ \\
& \theta\left(l_{1}-\xi\right) \varphi_{1}^{*}(\xi, \lambda)+\theta\left(\xi-l_{n}\right) \varphi_{n+1}^{*}(\xi, \lambda)
\end{aligned}
$$

$\left(a_{m}^{2} \frac{d^{2}}{d x^{2}}+\lambda^{2}\right) \varphi_{m}^{*}(x, \lambda)=0, x \in\left(l_{m}, l_{m+1}\right) ; m=1, \ldots, n+1$,

with the conjugation conditions

$$
\frac{1}{\Delta_{1, k}}\left[\alpha_{m 1}^{k} \frac{d}{d x}+\beta_{m 1}^{k}\right] \varphi_{k}^{*}=\frac{1}{\Delta_{2, k}}\left[\alpha_{m 2}^{k} \frac{d}{d x}+\beta_{m 2}^{k}\right] \varphi_{k+1}^{*}, x=l_{k},
$$

$$
\Delta_{i, k}=\operatorname{det}\left(\begin{array}{c}
\alpha_{1 i}^{k} \beta_{1 i}^{k} \\
\alpha_{2 i}^{k} \beta_{2 i}^{k}
\end{array}\right) k=1, \ldots, n ; i, m=1,2,
$$

on the boundary conditions

$\left.\varphi_{1}\right|_{x=-\infty}=0,\left.\varphi_{n+1}\right|_{x=\infty}=0$

Let for some $\lambda$ of the considered boundary value problems have nontrivial solutions $\varphi(x, \lambda), \varphi^{*}(x, \lambda)$, in this case the number $\lambda$ is called the eigenvalue, corresponding solutions $\varphi(x, \lambda), \varphi^{*}(x, \lambda)$ - is called the eigenfunctions of the direct and conjugate Sturm-Liouville problems, respectively. In the further we shall adhere to the following normalization of eigenfunctions:

$$
\varphi_{n+1}(x, \lambda)=e^{i a_{n+1}^{-1} x \lambda} \cdot \varphi_{n+1}^{*}(x, \lambda)=e^{-i a_{n+1}^{-1} x \lambda} .
$$

\section{Analogues of the system the Hermite functions on piecewise-homogeneous real axis}

Let define analogues of the system the Hermite functions on piecewise-homogeneous real axis:

$$
\begin{aligned}
& H_{j, n}(x)=\int_{-\infty}^{\infty} \varphi(x, \lambda) H_{j}\left(\frac{\lambda}{2 \sqrt{\tau}}\right) d \lambda, \\
& H_{j, n}^{*}(x)=\int_{-\infty}^{\infty} \varphi^{*}(x, \lambda) H_{j}(2 \sqrt{\tau} \lambda) d \lambda .
\end{aligned}
$$

where $H_{j}$ - the system of the classical orthogonal Hermite functions [1].

Lemma 1. Functions $H_{j, n}(x), H_{j, n}^{*}(x)$ form biorthogonal system of functions by piecewise-homogeneous real axis.

Proof. We have the equality:

$$
\begin{aligned}
& \int_{-\infty}^{\infty} H_{j, n}(x) H_{k, n}^{*}(x) d x= \\
& =\int_{-\infty}^{\infty}\left(\int_{-\infty}^{\infty} \varphi(x, \lambda) H_{j}(\lambda) d \lambda\right)\left(\int_{-\infty}^{\infty} \varphi^{*}(x, \beta) H_{k}(\beta) d \beta\right) d x .
\end{aligned}
$$

We change the integrals of places, we get:

is a solution of the system of separate differential equations 
$\int_{-\infty}^{\infty} H_{j, n}(x) H_{k, n}^{*}(x) d x=$

$=\int_{-\infty}^{\infty} H_{j}(\lambda)\left(\int_{-\infty}^{\infty} \varphi(x, \lambda)\left(\int_{-\infty}^{\infty} \varphi^{*}(x, \beta) H_{k}(\beta) d \beta\right) d x\right) d \lambda$.

On the decomposition theorem, we have:

$H_{k}(\lambda)=\int_{-\infty}^{\infty} \varphi(x, \lambda)\left(\int_{-\infty}^{\infty} \varphi^{*}(x, \beta) H_{k}(\beta) d \beta\right) d x$.

Consequently,

$\int_{-\infty}^{\infty} H_{j, n}(x) H_{k, n}^{*}(x) d x=\int_{-\infty}^{\infty} H_{j}(\lambda) H_{k}(\lambda) d \lambda=\delta_{j, k}$.

\section{Main result}

The problem of determining the initial distribution of the temperature field $f(x)$ mathematically leads to the separate system of integral equations:

$\sum_{s=1}^{n+1} \int_{l_{s}}^{l_{s+1}} H_{k s}(\tau, x, \xi) f_{s}(\xi) d \xi=u_{k}(\tau, x)$.

$k=1, \ldots, n+1$.

Method of transformation operators applicable to solving separate system of integral equations (8).

Theorem 1. If the function $u(\tau, x) \in S^{\prime}(R)$ and for her the condition

$e^{\tau^{2} \lambda}\left(1+\lambda^{2}\right)^{\alpha / 2} \tilde{u}(\tau, \lambda) \in L_{2}(R)$,

that the separate system of integral equations (8) has a unique solution $f(x) \in H_{2}^{\alpha}\left(I_{n}\right)$ (definition $H_{2}^{\alpha}\left(I_{n}\right)_{[6]) \text {, is }}$ according to the formula:

$f(x)=\frac{1}{\sqrt{\pi}} \sum_{j=0}^{\infty} \frac{D_{n}(u)}{2^{j} j !} H_{j, n}(x)$,

where

$$
D_{n}(u)=\frac{1}{2 \pi} \int_{-\infty}^{\infty}(i \lambda)^{j} \tilde{u}(\tau, \lambda) d \lambda .
$$

Proof . Let's apply the transformation operator $J^{-1}$ to separate system of integral equations (8). As a result come to a model integral equation (1). Let's apply the operator $J$ in both parts of the obtained equality (9); as a result, taking into account the continuity of the operator $J$, we find the unknown distribution of temperature:

$f(x)=\frac{1}{\sqrt{\pi}} \sum_{j=0}^{\infty} \frac{\hat{u}^{(j)}(0) \tau^{\frac{j}{2}}}{(2 \sqrt{\tau})^{n+1} j !} H_{j, n}(x)$

Let's calculate numbers $\hat{u}^{(j)}(0)$.

$\hat{u}^{(j)}(0)=\frac{1}{2 \pi} \int_{-\infty}^{\infty}(i \lambda)^{j}\left(\int_{-\infty}^{\infty} e^{-i \lambda \xi} \hat{u}(\xi) d \xi\right) d \lambda$,

from the definition of the operator conversion of $J$ the equality follows:

$$
\int_{-\infty}^{\infty} e^{-i \lambda \xi} \hat{u}(\xi) d \xi=\int_{-\infty}^{\infty} \varphi^{*}(\xi, \lambda) u(\xi) d \xi .
$$

Thus,

$\hat{u}^{(j)}(0)=\frac{1}{2 \pi} \int_{-\infty}^{\infty}(\mathrm{i} \lambda)^{\mathrm{j}} \tilde{u}(\tau, \lambda) d \lambda$.

\section{Power function with discontinuous coefficients and its application}

We consider the Fourier transform of the Delta function

$F[\delta(x)]=\int_{-\infty}^{\infty} e^{-i \lambda x} \delta(x) d x=1$,

consequently

$F^{-1}[1]=\frac{1}{2 \pi} \int_{-\infty}^{\infty} e^{-i \lambda x} d x=\delta(\lambda)$.

We find as a consequence

$F\left[x^{k}\right]=i^{k} \frac{d^{k}}{d \lambda^{k}}\left(\int_{-\infty}^{\infty} e^{-i \lambda x} d x\right)=2 \pi i^{k} \delta^{k}(\lambda)$

Let's define analog of the power function as follows

$x_{n}^{k}=(i)^{k} \frac{\partial^{k} \varphi(x, \lambda)}{\partial \lambda^{k}} \mid \lambda=0$.

We find equality from the definition of the transformation operators

$J\left[x^{k}\right]=x_{n}^{k} ; J^{-1}\left[x_{n}^{k}\right]=x^{k}$.

The last equality means that the power function with discontinuous coefficients is obtained by the action of the transformation operator to the power function.

Theorem 2. The ratio connects the generalized power function and differentiation 
$\frac{d^{2}}{d x^{2}} x_{n}^{k}=k(k-1) x_{n}^{k-2}$.

Proof. We have a chain of equalities

$\frac{d^{2}}{d x^{2}}\left(x_{n}^{k}\right)=\frac{d^{2}}{d x^{2}} J\left(x^{k}\right)=J\left(\frac{d^{2}}{d x^{2}} x^{k}\right)=$

$J\left(k(k-1) x^{k-2}\right)=k(k-1) x_{n}^{k-2}$

\section{Retrospective problem for the system of the diffusion equations}

Let's return to the solution of the separate system of integral equations (8) in space of the generalized functions $S^{\prime}$

$$
f(x)=\frac{1}{2 \pi} \int_{-\infty}^{\infty} e^{\lambda^{2} \tau} \varphi(x, \lambda) \int_{\infty}^{\infty} \varphi^{*}(x, \xi) u(\tau, \xi) d \xi d \lambda
$$

$k=1, \ldots, n+1$.

We will convert the found solution. An analogue of the Taylor series for the function $u(\tau, \xi)$ is of the form

$u(\tau, \xi)=\sum_{j=0}^{\infty} \frac{u_{j}(\tau)}{j !} x_{n}^{j}$

From decomposition theorem have

$u(\tau, x)=$

$=\frac{1}{2 \pi} \sum_{s=1}^{n+1} \int_{-\infty}^{\infty} \varphi(x, \lambda) \int_{l_{s}}^{l_{s+1}} \varphi_{s}^{*}(x, \xi) u_{s}(\tau, \xi) d \xi d \lambda$.

Let's find the decomposition of the generalized Taylor series for eigenfunction from definition of the generalized power function

$$
\varphi(x, \lambda)=\sum_{j=0}^{\infty} \frac{(i \lambda)^{j}}{j !} x_{n}^{j}
$$

Let's substitute this decomposition in a formula (11) and let's integrate term by term. We come to the formula (12) in which

$u_{j}(\tau)=\frac{1}{2 \pi} \sum_{s=1}^{n+1} \int_{-\infty}^{\infty}(i \lambda)^{j} \int_{l_{s}}^{l_{s+1}} \varphi_{s}^{*}(x, \xi) u_{s}(\tau, \xi) d \xi d \lambda$.

Let's substitute decomposition of eigenfunction $\varphi(x, \lambda)$ in the generalized power series in a formula (10) we will receive

$$
\begin{gathered}
f(x)=\frac{1}{2 \pi} \int_{-\infty}^{\infty} e^{\lambda^{2} \tau} \varphi(x, \lambda) \int_{\infty}^{\infty} \varphi^{*}(x, \xi) \sum_{j=0}^{\infty} \frac{u_{j}(\tau)}{j !} \xi_{n}^{j} d \xi d \lambda= \\
=\sum_{j=0}^{\infty} \frac{u_{j}(\tau)}{j !} \frac{1}{2 \pi} \int_{-\infty}^{\infty} e^{\lambda^{2} \tau} \varphi(x, \lambda) \int_{\infty}^{\infty} \varphi^{*}(\xi, \lambda) \xi_{n}^{j} d \xi d \lambda= \\
=\sum_{j=0}^{\infty} \frac{u_{j}(\tau)}{j !} \frac{1}{2 \pi} \int_{-\infty}^{\infty} e^{\lambda^{2} \tau} \varphi(x, \lambda) 2 \pi i^{j} \delta^{j}(\lambda) d \lambda=
\end{gathered}
$$

$=\sum_{j=0}^{\infty} \frac{u_{j}(\tau)(-1)^{j}}{j !} \int_{-\infty}^{\infty} \frac{\partial^{j}}{\partial \lambda^{j}}\left(e^{\lambda^{2} \tau} \varphi(x, \lambda)\right)^{j} \delta^{j}(\lambda) d \lambda=$

$=\sum_{j=0}^{\infty} \frac{u_{j}(\tau)}{j !} H_{j n}(x)$

where designation is accepted

$$
H_{j n}(x)=\left.(-i)^{j} \frac{\partial^{j}}{\partial \lambda^{j}}\left(e^{\lambda^{2} \tau} \varphi(x, \lambda)\right)\right|_{\lambda=0} .
$$

Thus, the solution of the retrospective problem (10) is obtained.

Remark 1. The generating function for the $H_{j n}(x)$ is the form $e^{-\lambda^{2} \tau} \varphi(x,-i \lambda)=\sum_{j=0}^{\infty} \frac{H_{j n}(x)}{j !} \lambda^{j}$.

Corollary 1. If to choose

$$
t=\frac{1}{2}, n=1, \varphi(x, \lambda)=e^{i \lambda x},
$$

then we obtain

$$
e^{-\frac{1}{2} \lambda^{2}} e^{\lambda x}=\sum_{j=0}^{\infty} \frac{H_{j 1}(x)}{j !} \lambda^{j},
$$

functions $H_{j 1}(x)$-are the classical Hermite polynomials.

\section{Retrospective problem for fractal systems of diffusion equations}

Retrospective problem for fractal system of diffusion equations in the space of generalized functions $S^{\prime}$ leads to the separatist system of integral equations:

$$
\begin{aligned}
f(x) & =\frac{1}{2 \pi} \int_{-\infty}^{\infty} E_{\alpha, 1}\left(\lambda^{2} \tau^{\alpha}\right) \varphi(x, \lambda) \int_{\infty}^{\infty} \varphi^{*}(x, \xi) u(\tau, \xi) d \xi d \lambda, \\
k & =1, \ldots, n+1,
\end{aligned}
$$

where $E_{\alpha, 1}(z)$ - the Mittag-Leffler function [9].

We get the solution of the fractal retrospective problem repeating reasoning's from paragraph VII.

$f(x)=\sum_{j=0}^{\infty} \frac{u_{j}(\tau)}{j !} H_{j n}(x)$,

where designation is accepted

$$
H_{j n}(x)=\left.(-i)^{j} \frac{\partial^{j}}{\partial \lambda^{j}}\left(E_{\alpha, 1}\left(\lambda^{2} \tau^{\alpha}\right) \varphi(x, \lambda)\right)\right|_{\lambda=0} .
$$

You can find the generating functions for $H_{j n}(x)$ :

$E_{\alpha, 1}\left(-\lambda^{2} \tau^{\alpha}\right) \varphi(x,-i \lambda)=\sum_{j=0}^{\infty} \frac{H_{j n}(x)}{j !} \lambda^{j}$.

We find the explicit expression for the functions $H_{j n}(x)$. We find decomposition of the left part of the formula (15) in the Taylor series 


$$
\begin{array}{cc}
E_{\alpha, 1}\left(-\lambda^{2} \tau^{\alpha}\right) \varphi(x,-i \lambda)=\sum_{k=0}^{\infty}(-1)^{k} \frac{\lambda^{2 k} \tau^{\alpha k}}{\Gamma(k \alpha+1)} \sum_{m=0}^{\infty} \frac{\lambda^{m}}{m !} x_{n}^{m}=\left.\frac{1}{2}(-i)^{j} \frac{\partial^{j}}{\partial \lambda^{j}}\left(e^{i \lambda(\tau+x)}+e^{i \lambda(\tau-x)}\right)\right|_{\lambda=0}= \\
=\sum_{j=0}^{\infty} \lambda^{j} \sum_{2 k+m=j}(-1)^{k} \frac{\tau^{\alpha k}}{\Gamma(k \alpha+1) m !} x_{n}^{m}= & =\frac{(\tau+x)^{j}+(\tau-x)^{j}}{2} .
\end{array}
$$$$
=\sum_{j=0}^{\infty} \lambda^{j} \sum_{k=0}^{\left[\frac{n}{2}\right]} \frac{(-1)^{k} \tau^{\alpha k} j !}{\Gamma(k \alpha+1)(j-2 k) !} x_{n}^{j-2 k} .
$$

We get the expression, comparing the two views $H_{j n}(x)$

In the end we find the solution of the Cauchy problem for the hyperbolic equation

$$
f(x)=\sum_{j=0}^{\infty} u^{j}(\tau, 0) \frac{(\tau+x)^{j}+(\tau-x)^{j}}{2 j !} .
$$

Thus,

$$
H_{j n}(x)=\sum_{k=0}^{\left[\frac{n}{2}\right]} \frac{(-1)^{k} \tau^{\alpha k} j !}{\Gamma(k \alpha+1)(j-2 k) !} x_{n}^{j-2 k} .
$$

$f(x)=\frac{u(\tau, \tau+x)+u(\tau, \tau-x)}{2}$.

If $n=1$, the formula takes the form

$$
H_{j 1}(x)=\sum_{k=0}^{\left[\frac{j}{2}\right]} \frac{(-1)^{k} \tau^{\alpha k} j !}{\Gamma(k \alpha+1)(j-2 k) !} x^{j-2 k} .
$$

Remark. If, as an example, take

$u(t, x)=g(x+t-\tau)+g(x-t+\tau)$,

Define a "fractal" generalization of the Hermite polynomials

$$
H_{j}(x)=\sum_{k=0}^{\left[\frac{j}{2}\right]} \frac{(-1)^{k} j !}{\Gamma(k \alpha+1)(j-2 k) !} x^{j-2 k},
$$

then the solution of the fractal retrospective problem has the form

$$
f(x)=\sum_{j=0}^{\infty} \frac{u_{j}(\tau) \tau^{\beta j}}{j !} H_{j}\left(\frac{x}{\tau^{\beta}}\right), \beta=\frac{\alpha}{2} .
$$

$u(\tau, x)=2 g(x)$ and, so,

$$
f(x)=g(x+\tau)+g(x-\tau)=u(0, x) .
$$

\section{The inverse dirichlet problem for a half-plane}

Solution of the inverse Dirichlet problem for the right half-plane has the form:

$f(y)=\operatorname{Re} \frac{1}{\pi} \int_{0}^{\infty} e^{\lambda l} e^{i \lambda y} \int_{-\infty}^{\infty} e^{-i \lambda \eta} u(l, \eta) d \eta d \lambda$.

Corollary 2. In the hyperbolic case $\alpha=2, n=1$ the solution of the retrospective problem has the form

Let's repeat the above reasoning. Let's receive expressions for analogues of Hermite polynomials:

$$
f(x)=\sum_{j=0}^{\infty} \frac{u_{j}(\tau) \tau^{j}}{j !} H_{j}\left(\frac{x}{\tau}\right), \beta=\frac{\alpha}{2} .
$$

$$
\begin{aligned}
& H_{j 1}(x)=\left.\operatorname{Re}(-i)^{j} \frac{\partial^{j}}{\partial \lambda^{j}}\left(e^{\lambda l} e^{i \lambda y}\right)\right|_{\lambda=0}= \\
& =\operatorname{Re}(-i)^{j}(l+i y)^{j}=\frac{(y+l i)^{j}+(y-l i)^{j}}{2} .
\end{aligned}
$$

$$
\begin{aligned}
H_{j}(x) & =\sum_{k=0}^{\left[\frac{j}{2}\right]} \frac{(-1)^{k} j !}{((2 k) !)(j-2 k) !} x^{j-2 k}=\sum_{k=0}^{\left[\frac{j}{2}\right]}(-1)^{k} C_{j}^{2 k} x^{j-2 k} \\
= & \frac{(1+x)^{j}+(1-x)^{j}}{2}
\end{aligned}
$$

Proof. We replace $\lambda$ for $i \lambda$ in formula (16) for the solution of the direct problem. As a result, we get the formula

$$
u(\tau, x)=\sum_{j=0}^{\infty} \frac{f_{j} \tau^{\beta j}}{j !} H_{j}^{*}\left(\frac{x}{\tau^{\beta}}\right), H_{j}^{*}(z)=i^{j} H_{j}(-i z) .
$$

We use the formula (15)

$$
H_{j 1}(x)=\left.(-i)^{j} \frac{\partial^{j}}{\partial \lambda^{j}}\left(\cos (\lambda \tau) e^{i \lambda x}\right)\right|_{\lambda=0}=
$$

As a result for the solution of the inverse Dirichlet problem we receive the representation in the form of the sum of the Taylor series:

$f(y)=\sum_{j=0}^{\infty} \frac{u^{j}(l, 0)}{j !} \frac{(y+l i)^{j}+(y-l i)^{j}}{2}$.

Corollary 3. If the function $f(y)$ admit continued with the real axis of the complex plane as a whole, the

$$
f(y)=\operatorname{Re} u(l, y+l i) .
$$

Example 2. Let $u(x, y)=(x-l)^{2}-y^{2}$, then $u(l, y)=-y^{2}$ therefore, we find 


$$
f(y)=\operatorname{Re}\left(-(y+l i)^{2}\right)=l^{2}+y^{2}=u(0, y) .
$$

\section{Conclusion}

In this article the formal solution of the retrospective problem is provided. The third aspect in determining the well-posed problem is not taken into account. Theorem of existence and uniqueness of solution are given. From the analysis of the formula (9): the solution of the retrospective heat problem with discontinuous coefficients is received by replacement in the final result of the Hermite functions [1] on the Hermite functions with discontinuous coefficients, defined in the article. The derivatives $\hat{u}^{j}(0)$ are necessary to replace on $D_{n}(u)$. The noticed analogy allows hoping on the possibility of obtaining the solution of problems of mathematical physics in which the Hermite functions with discontinuous coefficients.

\section{References}

[1] F.M. Mors, G. Fishbah, Methods of theoretical physics, 1958.

[2] Yaremko, O.E. Matrix integral Fourier transforms for problems with discontinuous coefficients and transformation operators (2007) Doklady Mathematics, 76 (3), pp. 323-325.

[3] O.M. Alifanov, Inverse problems of heat exchange, $\mathrm{M}$, 1988, p. 279.

[4] O.M. Alifanov, B.A. Artyukhin, S.V. Rumyancev, The extreme methods of solution of ill-posed problems, M, 1988, p. 288.

[5] J.V. Beck, V. Blackwell, C.R. Clair, Inverse Heat Conduction. Ill-Posed Problems , M, 1989, p. 312.

[6] V.K. Ivanov, V.V. Vasin, V.P. Tanana, Theory of linear illposed problems and its applications, M, 1978, p. 206.

[7] M.M. Lavrentev, Some ill-posed problems of mathematical physics, Novosibirsk, AN SSSR,1962, p. 92.

[8] A.N. Tikhonov, V. Ya. Arsenin, Methods of solution of illposed problems, M,1979, p. 288.

[9] M.M. Dzhrbashyan, Integral Transforms and Representations of Functions in the Complex Domain, M, 1966. 